\title{
Optimization of Quantum Trajectories Driven by Strong-Field Waveforms
}

\author{
S. Haessler, T. Balčiunas, G. Fan, G. Andriukaitis, A. Pugžlys, and A. Baltuška \\ Photonics Institute, Vienna University of Technology, Gußhausstraße 27/387, 1040 Vienna, Austria
}

T. Witting, R. Squibb, ${ }^{\dagger}$ A. Zaï, J. W. G. Tisch, and J. P. Marangos

Blackett Laboratory, Imperial College, London SW7 2AZ, United Kingdom

L. E. Chipperfield

Max Born Institute, Max-Born-Straße 2 A, 12489 Berlin, Germany

(Received 17 January 2014; published 19 May 2014)

\begin{abstract}
Quasifree field-driven electron trajectories are a key element of strong-field dynamics. Upon recollision with the parent ion, the energy transferred from the field to the electron may be released as attosecondduration extreme ultaviolet emission in the process of high-harmonic generation. The conventional sinusoidal driver fields set limitations on the maximum value of this energy transfer and the efficient return of the launched electron trajectories. It has been predicted that these limits can be significantly exceeded by an appropriately ramped-up cycle shape [L. E. Chipperfield et al., Phys. Rev. Lett. 102, 063003 (2009)]. Here, we present an experimental realization of similar cycle-shaped waveforms and demonstrate control of the high-harmonic generation process on the single-atom quantum level via attosecond steering of the electron trajectories. With our improved optical cycles, we boost the field ionization launching the electron trajectories, increase the subsequent field-to-electron energy transfer, and reduce the trajectory duration. We demonstrate, in realistic experimental conditions, 2 orders of magnitude enhancement of the generated extreme ultraviolet flux together with an increased spectral extension. This application, which is only one example of what can be achieved with cycle-shaped high-field light waves, has significant implications for attosecond spectroscopy and molecular self-probing.
\end{abstract}

DOI: 10.1103/PhysRevX.4.021028

\section{INTRODUCTION}

Controlling the shape of the driving laser waveform allows direct steering of the quasifree electron trajectories at the heart of strong-field phenomena [1,2]. Technically, this requires the generation of powerful phase-locked morethan-octave spanning spectra, which until recently $[3,4]$ had only been possible by combining a fundamental with one of its optical harmonics, typically the second. Early in the development of high-harmonic generation (HHG) light sources, it was shown that such two-color fields can enhance the spectral content and brightness of the generated extreme ultraviolet (XUV) emission [5]. The appearance of even as well as odd order harmonics reflects the additional periodicities in the electric field waveform when a fundamental field and its second harmonic are employed.

\footnotetext{
* Corresponding author. stefan.haessler@tuwien.ac.at

'Present address: Department of Physics and Astronomy, Uppsala University, SE-751 20 Uppsala, Sweden.
}

Published by the American Physical Society under the terms of the Creative Commons Attribution 3.0 License. Further distribution of this work must maintain attribution to the author(s) and the published article's title, journal citation, and DOI.
Subject Areas: Optics, Photonics, Quantum Physics

For the case of a very weak second harmonic, the additional field interaction has been found to act as a phase gate in HHG that can be used to extract the return [6] or ionization instants [7] of the recolliding trajectories. Stronger secondharmonic fields have been used in optical gating schemes [8] for isolated attosecond pulse generation $[9,10]$. Several factors have been shown to contribute to enhanced brightness in the HHG spectrum: enhanced tunnel ionization at the "right" instants when recolliding trajectories are launched [11,12], the alteration of the phases [13] of the quantum trajectories responsible for a given frequency high-harmonic field, or their coalescence in a spectral caustic [14]. Many examples of this have been experimentally studied, including both combinations of a fundamental with an optical harmonic, where relative phase control is possible, and schemes involving incommensurate frequencies with no possibility for systematic phase control [15]. The most substantial efficiency enhancements have been reported for orthogonally polarized two-color combinations of a fundamental with its second harmonic $[11,16]$. Since in such fields the conditions for the recollision of trajectories have to be satisfied in two independent dimensions, the selection of ionization instants that lead to harmonic emission is more severe than for parallel polarizations. This limits the spectral range over which enhancement of 
HHG can be achieved, e.g., in Ref. [11] to the lowest-order plateau harmonics. Use of orthogonally polarized fields also provides a control over the angle and displacement of the returning electron trajectories [17], which has been shown to be of utility in HHG self-probing of the source atom or molecule [18], and in selection of the dominant trajectory class (short or long) [19]. To gain the full benefit of both enhanced field ionization and steering of the electron return for HHG efficiency enhancement, it is, however, preferable to use multifrequency fields with parallel polarization.

While even subtle modifications of the driving waveform can yield significant control over HHG [20], the theoretical work on the "perfect wave for HHG" [21] showed that a fully optimized waveform will deviate drastically from a sinusoidal shape: a temporally confined ionization event coupled to an electric field ramp to ensure the required rapid return of most trajectories will optimize HHG in terms of highest cutoff and efficiency. Despite the numerous successes of two-color drive fields, it is clear that with only two colors the scope for waveform (Fourier) shaping remains rather limited and the efficient synthesis of a waveform approximating to the "perfect wave" ideal requires more frequency components. The obvious next step is to use three phase-locked fields with control of the two independent phase delays. Two extensions in this direction have recently been reported. In one case [22], a combination of an 800-nm fundamental with its second and third harmonic allowed the control of the electron trajectory phases for a particular return energy to improve intrapulse phase matching and enhance the generation of a single harmonic. However, the two relative field phases were not independently controlled nor was the enhancement demonstrated over a broad spectral range as required for attosecond pulse synthesis. In the other case, three broadband field components [3], generated through selfphase modulation in a hollow-core fiber and then separated, individually manipulated, and recombined, were used for the subfemtosecond confinement of field ionization to a single field crest. While the synthesized field has been used for generation of isolated attosecond pulses through HHG, no enhancement or control of this generation via the delays of the three field components has been reported. The scaling to higher pulse energies is hampered by the limitations of hollow-core fibers and would thus require costly separate parametric amplification for each color component. Note that in that method [3], each field component already has a very large bandwidth, so the resulting field waveform is hard to analyze in terms of standard Fourier synthesis.

Here, we apply shaped optical cycles, synthesized from three discrete spectral bands covering 1.6 octaves, to the complex optimization task of efficient launch, acceleration, and return of the electron trajectories in HHG. This addresses a fundamental bottleneck in HHG driven by a sinusoidal driver of wavelength $\lambda$ and intensity $I$, which determine the highest achievable electron energy at recollision, 3.2 $U_{p}$, where $U_{p} \propto I \lambda^{2}$. The laser intensity that can be used is limited to a narrow range due to the combined effect of the exponentially growing ionization rate and saturation (near unity ionization probability). Increasing the wavelength leads to a drop $\propto \lambda^{-6}$ of the conversion efficiency [23] as a consequence of increased trajectory
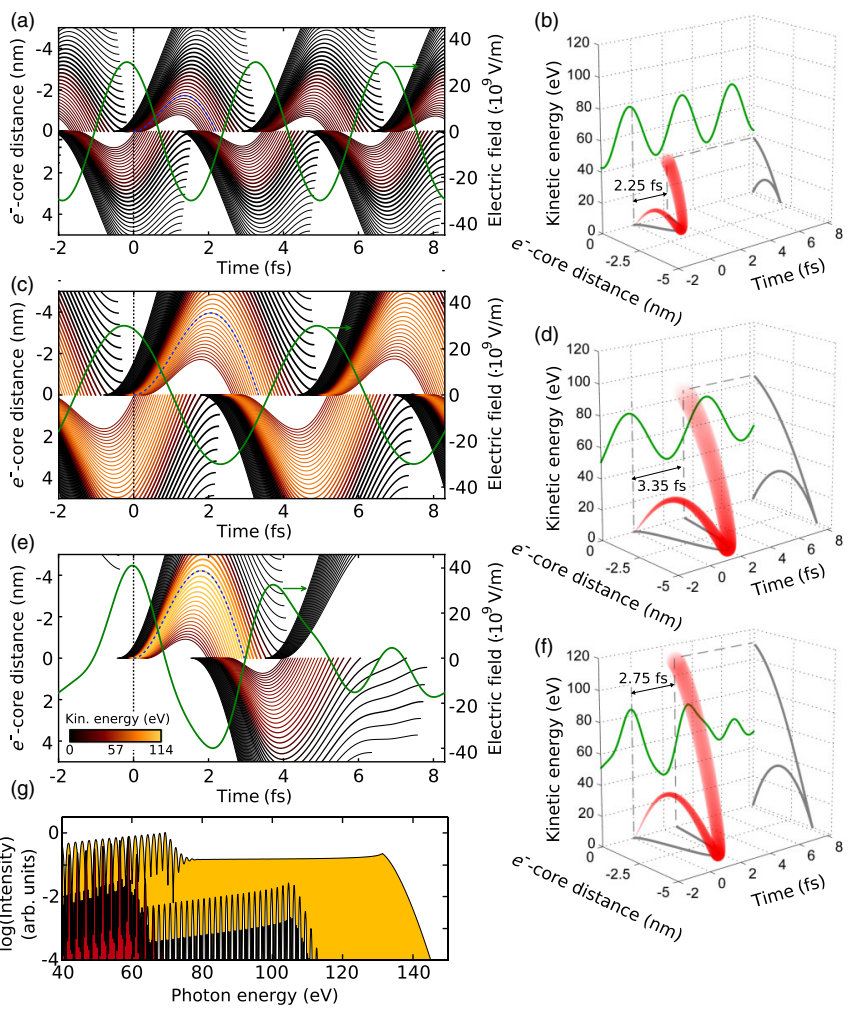

FIG. 1. (a)-(f) Classical electron trajectories, with driving electric field shown by the green line, (g) HHG spectra. Both single-color drivers [1.03 $\mu \mathrm{m}$ (a),(b) and $1.545 \mu \mathrm{m}$ (c),(d)] have an intensity of $1.2 \times 10^{14} \mathrm{~W} \mathrm{~cm}^{-2}$. The three-color waveform [(e),(f), combination of $1.03,0.515$, and $1.545 \mu \mathrm{m}$ with relative intensities as in our experiments [Fig. 6(c)] and optimal phase delays (see also Ref. [24])] has the same fluence within its 10.3-fs period as the single-color waves. In (a), (c), and (e), the classical electron trajectories are colored according to their recollision energy [the color bar in (c) is also valid for (a) and (b), nonrecolliding trajectories are black]. A thicker line indicates a higher tunneling rate at the ionization instant (normalized in each panel). The cutoff trajectory with the highest recollision energy is marked by the dashed blue line, and its ionization instant, $t=0$, is marked by the vertical dotted line. This cutoff trajectory is again shown in (b), (d), and (f) by points with radii that linearly increase with excursion time, symbolizing wave packet spreading. The more efficient acceleration in the three-color field is apparent. Panel (d) shows the corresponding single-atom HHG spectra (red for $1.03 \mu \mathrm{m}$, white for $1.545 \mu \mathrm{m}$, yellow for the three-color driver) calculated for the ionization potential of argon $(15.76 \mathrm{eV})$ over a 10.3 -fs time window and including the short-trajectory class only [24]. 
excursion duration, $\propto \lambda$, which results in additional quantum wave packet spreading and so reduces the recollision amplitude (see Fig. 1). For some applications, phase matching in long high-pressure media can compensate for this loss [25]. As illustrated in Figs. 1(a) and 1(c), with sinusoidal driver waves a majority of the continuum electron wave packet does not return to the core and the cutoff trajectory is launched with a rather low tunneling rate. Figures 1(e) and 1(f) show that the synthesis of shaped optical cycles following the principles laid out by the "perfect wave for HHG" [21] would enhance the singleatom quantum dipole and, therefore, be an important step towards the removal of the above-mentioned bottleneck. With the tailored waveform, the highest recollision energies are achieved, the corresponding trajectories are launched with the highest tunneling rate, and the cutoff excursion duration is shortened. Nonrecolliding trajectories that do not contribute to HHG but only to detrimental ionization are much weaker. As shown in Fig. 1(g), this optimization should lead, in the single-atom emission, to a large cutoff extension as compared to the single-color near-IR driver (in our case, $1030 \mathrm{~nm}$ ), or a dramatic efficiency increase as compared to the IR driver (in our case, $1545 \mathrm{~nm}$ ). Here, we realize such optimized three-color waveforms and demonstrate the efficacy of the "perfect wave" concept even in realistic experimental conditions where macroscopic propagation effects are significant.

\section{WAVEFORM SHAPING SETUP}

We coherently combine three discrete color components with parallel polarizations: $\lambda_{1}=1030 \mathrm{~nm}$ from a $1-\mathrm{kHz}$ carrier-envelope phase (CEP-)locked Yb-based femtosecond laser amplifier, its second harmonic $\lambda_{2}=515 \mathrm{~nm}$, and $\lambda_{3}=1545 \mathrm{~nm}$, the signal wave from a white-light-seeded optical parametric amplifier (OPA), pumped by the 1030nm laser (see Ref. [24] for details).

In the well-proven combination of the optical harmonic with the fundamental, the relative phase delay remains locked independently of the CEP $\phi_{\mathrm{CEP}}$ of the fundamental. This is because, in the generation of the $n$th optical harmonic, the complete phase factor of the fundamental is multiplied by $n$, so that a CEP fluctuation shifts both the fundamental and its harmonic wave by the same phase delay $\phi_{\mathrm{CEP}} \lambda_{1}(2 \pi c)^{-1}=n \phi_{\mathrm{CEP}}\left(\lambda_{1} / n\right)(2 \pi c)^{-1}$. Adding more optical harmonics becomes increasingly difficult as the conversion efficiency strongly decreases with order. Furthermore, the frequency up-conversion rapidly reaches into the vacuum ultraviolet spectral region, which would create practical complications such as absorption in air and multiphoton ionization of the target medium.

In contrast, frequency-down-converted color components, created by OPA, can be generated with high conversion efficiency and reach towards the mid-IR spectral region, which is highly advantageous for strongfield interactions. In white-light-seeded OPA, the signal (wavelength $\lambda_{s}=\lambda_{1} / m, m<1$ ) "inherits" the CEP from the pump beam $\left(\lambda_{1}\right)$, while the idler $\left[\lambda_{i}=\lambda_{1} /(1-m)\right]$ has a passively stable CEP [26]. Thus, when the pump-laser CEP fluctuates, the idler's phase delay does not change, while the pump and signal shift by different phase delays: $\phi_{\mathrm{CEP}} \lambda_{1}(2 \pi c)^{-1} \neq \phi_{\mathrm{CEP}}\left(\lambda_{1} / m\right)(2 \pi c)^{-1}$. In our case, the active CEP locking of the Yb-based pump laser ensures stable and controllable relative phase delays of all color components. While this principle has been recognized before [4], here we report its first realization at sufficient power for driving HHG. While in this proof-of-concept work we do not yet use the phase-locked 3090-nm idler, which is also generated in our OPA, the extendability of our scheme by this additional color component, as well as by optical harmonics of the signal wave or additional OPA with different down-conversion factor $m$, is straightforward. The same is valid for the up-scaling of pulse energies and average power.

Our waveform synthesizer is schematically shown in Fig. 2. Using dichroic optics, part of the pump laser pulses $\left(\lambda_{1}\right)$ are split off the laser output before the OPA. The second harmonic at $\lambda_{2}=0.5 \lambda_{1}=515 \mathrm{~nm}$ is generated by frequency doubling a small part of this beam using a thin $(0.5 \mathrm{~mm}) \mathrm{BBO}$ crystal. The polarizations of the second harmonic and the fundamental are matched using a multicolor wave plate ( $\lambda$ for $515 \mathrm{~nm}, \lambda / 2$ for $1030 \mathrm{~nm}$ ), which also matches the polarizations to that of the OPA signal wave. The group velocity delay between the pump wave and its second harmonic is compensated by inserting an $\approx 1.5$-mm-thick calcite plate. The fine-tuning of the phase delay $\tau_{2}=\phi_{2} \lambda_{2}(2 \pi c)^{-1}$ between the fundamental $1030-\mathrm{nm}$ wave and its 515 -nm second harmonic is done by rotating a thin $(1 \mathrm{~mm})$ fused silica plate.

The white-light seed for the OPA is generated in a sapphire plate $(1 \mathrm{~mm})$. The OPA consists of three stages with type-II phase matching in KTP crystals and is tuned to amplify a signal wave at $\lambda_{3}=(3 / 2) \lambda_{1}=1545 \mathrm{~nm}$, such

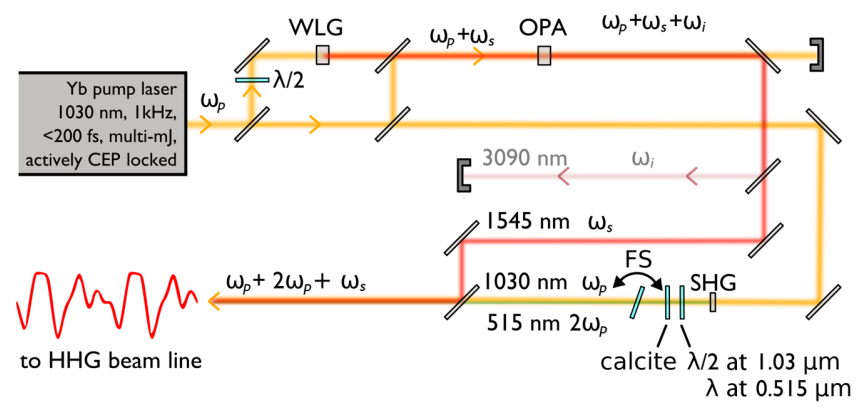

FIG. 2. Layout of the waveform synthesizer. A CEP-stabilized, multi-mJ, diode-pumped femtosecond Yb-based pump laser and a collinear white-light-seeded three-stage OPA are followed by a multicolor interferometer with subcycle stability and a secondharmonic generation (SHG) crystal in the 1- $\mu \mathrm{m}$ arm (see Ref. [24] for more details). White-light generation (WLG); fused silica (FS). 
that the idler wave is at $3090 \mathrm{~nm}$ (chosen to be the longest possible idler wavelength for which KTP is still transparent). An interference filter after the first OPA stage narrows the bandwidth around $1545 \mathrm{~nm}$, so that the final amplified signal pulse is nearly as long (170 fs) as the pump pulses and, most importantly, is close to Fourier limited. The signal wave is separated from the OPA output and then combined with the fundamental and its second harmonic via dichroic mirrors. The phase delay $\tau_{3}=$ $\phi_{\mathrm{CEP}}\left(\lambda_{3}-\lambda_{1}\right)(2 \pi c)^{-1}$ of the 1545 -nm signal wave relative to the 1030-nm fundamental wave is scanned by varying the CEP locking point of the pump laser amplifier slow loop.

Superposing our three color components with controlled phase delays, $\tau_{2}$ and $\tau_{3}$, we realize a Fourier synthesis of optical cycles, recurring with a 10.3 -fs period under the envelope of an $\approx 180$-fs laser pulse with $>0.5 \mathrm{~mJ}$ total energy. The main source of rapid phase jitter affects $\tau_{3}$ and is due to the 0.95 -rad rms jitter of $\phi_{\mathrm{CEP}}$. In high-intensity conditions with significant HHG medium ionization (such as in Fig. 6), the free-electron dispersion will lead to additional non-negligible averaging over both $\tau_{2}$ and $\tau_{3}$ in the HHG target-without, however, becoming prohibitive of effective HHG control via the driving waveform. See Ref. [24] for a discussion of the different factors that affect the waveform stability in the macroscopic HHG target. The synthesized waveforms are then focused $(\approx f / 15)$ about one Rayleigh length $(\approx 1 \mathrm{~mm})$ before a 0.8 -mm-long argonfilled gas cell. A $100-\mathrm{nm} \mathrm{Al}$ filter downstream transmits XUV radiation between 15.5 and $72.5 \mathrm{eV}$. This high-order harmonic emission is measured using a flat-field XUV spectrometer.

\section{SUBCYCLE, SINGLE-ATOM HHG CONTROL}

In order to study how the optical cycle shape governs the HHG emission, we set the peak intensities of the three color components at the HHG medium to $3 \times 10^{13} \mathrm{~W} \mathrm{~cm}^{-2} \quad(1030 \mathrm{~nm}), \quad 0.25 \times 10^{13} \mathrm{~W} \mathrm{~cm}^{-2}$ $(515 \mathrm{~nm})$, and $1.8 \times 10^{13} \mathrm{~W} \mathrm{~cm}^{-2}(1545 \mathrm{~nm})$, and measure the HHG spectra while scanning the phase delays $\tau_{2}\left(\phi_{2}\right)$ and $\tau_{3}\left(\phi_{\mathrm{CEP}}\right)$. To more clearly reveal the impact of the optical cycle shaping on the trajectories of the recolliding continuum electron wave packet (REWP), we normalize the measured HHG spectra by the squared recombination dipole matrix element for argon [24,27,28] (see Fig. 3). Furthermore, in order to highlight the fast subcycle dynamics, we filter out the dense $0.4-\mathrm{eV}$ harmonic peak modulation corresponding to the 10.3 -fs period of our waveforms [see Fig. 3(b)]. The result can be considered as a REWP spectrum generated within a single optical cycle of the driving wave.

Figure 4 shows these data as a function of the laser CEP $\phi_{\text {CEP }}$ for ten values of $\phi_{2}$ covering a $2 \pi$ range, together with REWP intensities simulated with the Lewenstein model
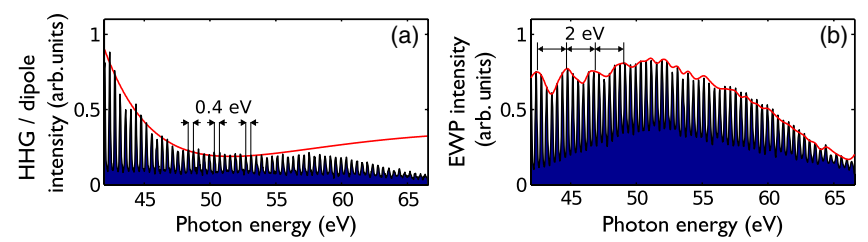

FIG. 3. (a) Measured HHG spectrum with $0.4-\mathrm{eV}$ harmonic peak spacing (blue area), taken out of the spectrogram shown in Fig. 4(a) for $\phi_{2}=1.19 \pi$ and $\phi_{\mathrm{CEP}}=2.6 \pi$. The red line shows the squared recombination dipole matrix element for argon, calculated by Fabre for Ref. [27]. (b) The same HHG spectrum divided by the squared recombination dipole matrix element gives the corresponding REWP spectrum. The red line shows the slowly varying spectral envelope, found by performing a peak search for the harmonic peaks and subsequent cubic spline interpolation.

[29] using the quantum path analysis [24,30]. These simulations are performed for a single atom and a single 10.3 -fs-long optical cycle and do not aim at reproducing in detail the experimental data (see Ref. [24] for further discussion of the simulations and their limits). Rather, they provide a reference for qualitative structures in the spectrograms (photon energy versus $\phi_{\mathrm{CEP}}$ ) as signatures of trajectory steering at the subcycle, single-atom level. We find good agreement of the overall structures with those observed experimentally for all $\phi_{2}$ values. This includes the slant of the dominant modulation with $\phi_{\text {CEP }}$ and the "phase jump" of this modulation between a lower and higher photon energy band for some $\phi_{2}$ values [see Figs. 4(a), 4(b), 4(f), 4(g), 4(j)]. The spectral modulation appearing for some $\phi_{2}$ values at photon energies below $\approx 55 \mathrm{eV}$ [see also Fig. 3(b)] has the same $\approx 2 \mathrm{eV}$ period in the experimental and simulated spectrograms. Our calculated quantum trajectories show that it corresponds to the interference of two recollision events per optical period, separated by $\approx 2$ fs [see, e.g., the two events in Figs. 5(a) and 5(b)]. There is no such modulation at the highest photon energies since these are produced only in a single recollision per optical cycle. The observed agreement is strong evidence that, despite experimental phase delay jitter [24], we control the HHG process on the single-atom level by directly steering electron quantum trajectories with our shaped optical cycles on the attosecond time scale.

The composite waveforms used in this work have the potential to significantly enhance HHG. As is visible in Fig. 4, the waveform corresponding to $\phi_{2}=1.19 \pi$ and $\phi_{\mathrm{CEP}}=1.9 \pi$ leads to good efficiency at high photon energies. Figure 5 shows the ionization and recollision instants of the quantum trajectories calculated for this waveform as well as the contribution of each trajectory to the HHG emission [24]. Only a single recollision event leads to the emission of photons above $45 \mathrm{eV}$. This is because the available driving field energy is concentrated to produce essentially two strong field crests per 10.3-fs 

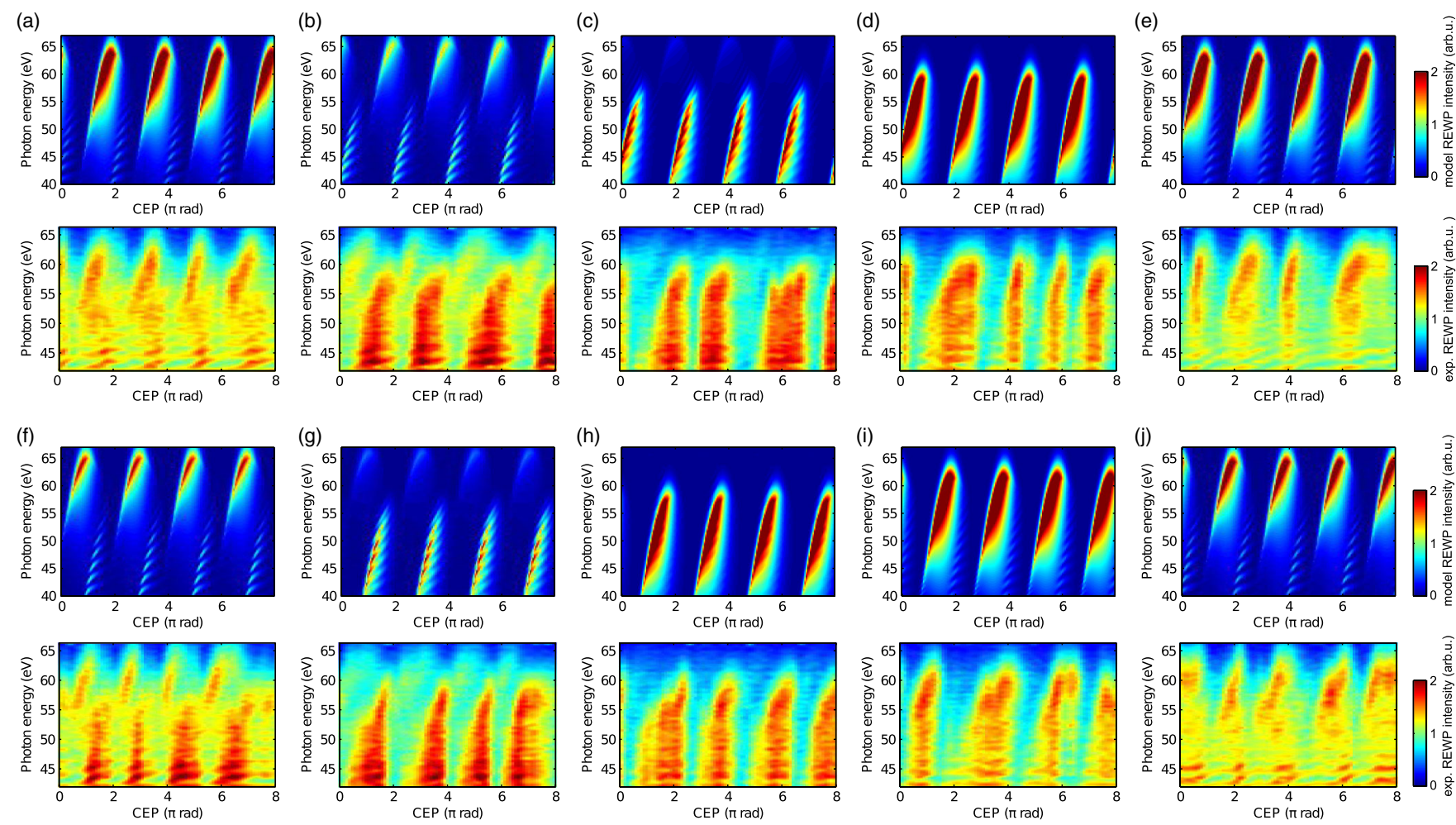

FIG. 4. (a)-(j) Simulated (upper) and measured (lower) REWP spectrograms for $\phi_{2}=1.19 \pi, 1.42 \pi, 1.65 \pi, 1.88 \pi, 0.11 \pi, 0.34 \pi$, $0.57 \pi, 0.80 \pi, 1.03 \pi$, and $1.26 \pi$. The simulations were performed for a single 10.3 -fs-long optical cycle using the same intensities of the three color components as estimated for the measurements. Only the short trajectory family has been included [24], since under the most commonly encountered experimental conditions this will be best phase matched in a macroscopic medium. The experimental $\phi_{\mathrm{CEP}}$ values have an unknown offset, which could slip between scans for different $\phi_{2}$. Note that in the low-ionization conditions of these experiments, $\phi_{2}$ may be considered as jitter-free, whereas $\phi_{\mathrm{CEP}}$ is averaged over $\geq 1 \mathrm{rad}$ [24]. Slow $(\sim 1 \mathrm{~Hz}) \tau_{3}$ jitter is responsible for deviations from the precise $2 \pi$ periodicity with $\phi_{\mathrm{CEP}}$ observed in some measured spectrograms [24]. HHG spectra were measured for $\pi / 10$ steps of $\phi_{\text {CEP }}$ averaging each time over 100 shots.

optical period, which can efficiently drive HHG. In a single-color wave with the same fluence per 10.3-fs time window, the attainable peak field strengths would always be lower, leading to reduced HHG efficiency. In the synthesized waveform, the high-energy trajectories are launched by the strongest field crest, and as shown in Fig. 5(c), the field crest maximum is very efficiently used for launching the recolliding trajectories for HHG. In contrast to sinusoidal driving fields, where all recolliding trajectories are launched after the field crest peak, the asymmetry between the ionizing and subsequent accelerating field crest in the synthesized waveform shifts the ionization instants to around the field crest maximum (similar to the ionization enhancement in Ref. [12]). The waveform after the ionization instants leads to an improved energy transfer from the driving field to the accelerated electron and thus enhances the recollision energies and thus the attainable HHG photon energy cutoff. The synthesized waveform leads to relatively short excursion durations of $<2.75 \mathrm{fs}$ for the relevant trajectories [for a single-color driver of, e.g., 1545-nm wavelength, the excursion duration for the cutoff trajectory would be significantly longer
(3.35 fs), without, however, leading to higher recollision energies]. This shape, a strong and sharp ionizing field crest followed by a ramped-up longer field crest, is reminiscent of that described for the "perfect wave" for HHG [21]. Our quantum-path analysis thus confirms the enhancement principles already highlighted by the classical trajectories shown in Figs. 1(e) and 1(f), which remain in effect even when the relative fluences of the color components are somewhat different.

For the quantitative question, how much enhancement will one get in the laboratory in a given spectral region?, we may get rough expectations from our model calculations, but a real answer requires either much more involved macroscopic simulations for the full multicolor laser pulses or, better yet, experiments such as those reported in the following.

\section{XUV FLUX ENHANCEMENT}

The experimental HHG data shown in Fig. 4 were obtained under conditions of fairly low ionization probabilities far from saturation. To demonstrate the full benefit 


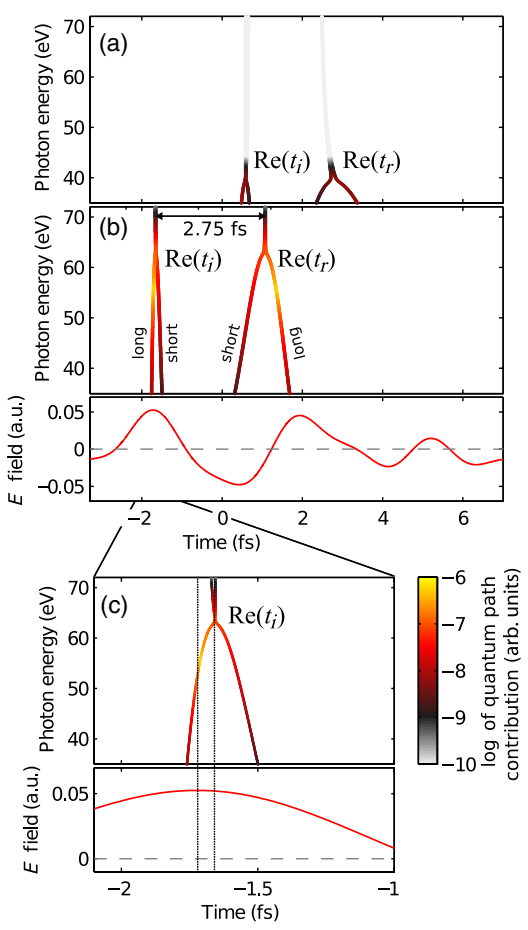

FIG. 5. Results of the saddle point analysis of the Lewenstein model simulations for the parameters of Fig. 2 with $\phi_{2}=1.19 \pi$ and $\phi_{\mathrm{CEP}}=1.9 \pi$, shown together with the driving waveform on the same time axis. In (a) and (b), a full optical cycle of the driving waveform is covered. Two recollision events corresponding to HHG emission above $35 \mathrm{eV}$ occur, shown separately in (a) and (b). Each event is represented by a matching pair of arches, the left and right of which represent the real parts of the ionization and recollision instants of the quantum trajectories, respectively. The left and right branches of the arches of ionization instants, joining at the spectral cutoff, correspond to the long and short trajectory class; the same applies vice versa to the arches of recollision instants. The data points are color coded, showing on logarithmic scale the contribution of the corresponding quantum path to the emitted HHG intensity. Panel (c) shows a zoom onto the ionization instants. The ionizing field strength launching the cutoff trajectories (at right vertical dashed line) is still $99 \%$ of its peak value (at left vertical dashed line). While the long trajectories give a higher contribution in this single-atom calculation, phase matching in a macroscopic medium usually leads to a clear dominance of the short-trajectory contribution. Atomic units (a.u.).

of our shaped optical cycles for the cutoff and efficiency enhancement of $\mathrm{HHG}$, we repeated our experiments at higher driving intensity and thus in conditions of high practical relevance close to saturation. This implies a reduced XUV phase-matching bandwidth and potentially some reshaping of the driving waveforms during propagation in the HHG medium. We also increased the relative strength of the 1545-nm component to further approach the optimal waveform theoretically predicted for our three color components [31]. The peak intensities of the three color components in the HHG medium were set to approximately $5.7 \times 10^{13} \mathrm{~W} \mathrm{~cm}^{-2}(1030 \mathrm{~nm})$,

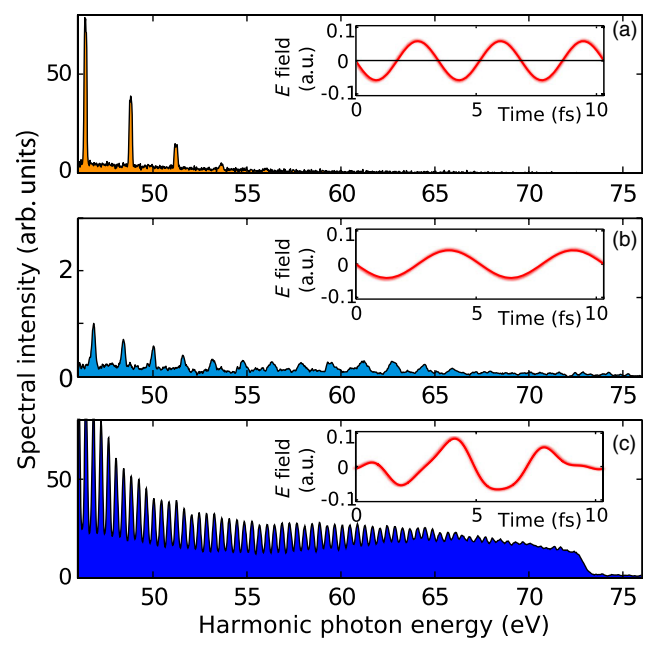

FIG. 6. Experimental HHG spectra generated in argon with different driver waves, shown in the insets. The spectral intensities are given on the same scale and are thus directly comparable - note, however, the different ordinate axis in (b). (a) $1030-\mathrm{nm}$ driver with the same $0.54-\mathrm{mJ}$ pulse energy as the three-color pulses, leading to the onset of saturation in HHG [24]. (b) 1545-nm driver with the full OPA output pulse energy $(0.35 \mathrm{~mJ})$. (c) Chosen three-color driver $\left(\phi_{2}=1.8 \pi\right.$ and $\left.\phi_{\mathrm{CEP}}=0.3 \pi\right)$ giving the highest HHG signal between $60 \mathrm{eV}$ and the Al-filter absorption edge. Atomic units (a.u.).

$0.3 \times 10^{13} \mathrm{~W} \mathrm{~cm}^{-2} \quad(515 \mathrm{~nm})$, and $6 \times 10^{13} \mathrm{~W} \mathrm{~cm}^{-2}$ $(1545 \mathrm{~nm})$. We compare the HHG spectra obtained with our shaped optical cycles to HHG with single-color drivers of 1545 and $1030 \mathrm{~nm}$ wavelength.

For this experimental study, summarized in Fig. 6, we do not compare the different driver waves at constant fluence during a given period (as in the simulations of Fig. 1), but rather at their respective practical limits in our experimental setup, which set the experimentally relevant benchmarks. All experimental conditions except for the pulse energies were left exactly the same for the single-color benchmarks as for the synthesized waveforms. The high-energy 1030nm pump laser easily drives HHG into saturation [24], which thus sets the practical limit. HHG saturation by the OPA output at $1545 \mathrm{~nm}$ was not achieved due to energy restrictions and wavelength scaling. We have optimized the OPA for highest conversion efficiency and output beam quality, so using the full OPA output sets the practical limit for HHG. For the synthesized waveforms, we performed a similar scan [24] as shown in Fig. 4 and then chose the waveform $\left(\phi_{2}=1.8 \pi\right.$ and $\left.\phi_{\mathrm{CEP}}=0.3 \pi\right)$ that produced the highest HHG signal between 60 and $72.5 \mathrm{eV}$ (Al-filter edge). Note that this is not the same waveform as shown in Fig. 1(c), which would maximize the HHG efficiency above $100 \mathrm{eV}$ and thus beyond the experimentally detected spectral range.

Figure 6 shows strong enhancement of HHG with the synthesized optical cycles. While a fairly high spectral cutoff $(66 \mathrm{eV})$ is achieved with the 1545-nm 
short-wavelength-IR (SWIR) driver, the HHG flux is very low. On the other hand, in HHG driven to the onset of saturation by the 1030-nm pump laser, we cannot shift the cutoff beyond $60 \mathrm{eV}$. Figures 6(a) and 6(b) thus clearly demonstrate the limitations of sinusoidal driver waves. In comparison, the synthesized optimal waveform generates a HHG spectrum that unites high spectral intensities with a cutoff well beyond the saturation limit of the efficient nearIR driver. In terms of XUV spectral intensity, the threecolor waveform is on par with the near-IR driver and leads to a $>80$ enhancement factor compared to the SWIR driver. The denser harmonic comb spacing leads to even greater enhancement in the integral XUV flux (measured factor $>140$ in the $55-65 \mathrm{eV}$ range). According to our simulations for the synthesized waveform [24], the dominant recollision events occur once per 10.3 fs (it would take a $6.2-\mu \mathrm{m}$ single-color driver to realize the same periodicity), as opposed to once per 2.6 fs in the SWIR case. Consequently, we would expect a several hundred times enhancement in the flux per attosecond burst and, thus, there are favorable implications to future sources of high-energy (isolated) attosecond pulses.

\section{CONCLUSIONS AND OUTLOOK}

We conclude that under realistic experimental conditions, the capability of quantum trajectory engineering via the shaping of the driving optical cycles provides a powerful tool to significantly enhance HHG. By generating waveforms that follow the guidelines of the theoretically proposed "perfect wave" for HHG [21], we avoid the cutoff-versus-efficiency dilemma [23] of HHG with sinusoidal driver waves.

The HHG driving performance of our waveforms goes well beyond what has been reached before. In the two-color method of Ref. [11], the stringent selectivity for the return of electron trajectories required a driving intensity of $\sim 10^{15} \mathrm{~W} \mathrm{~cm}^{-2}$ to demonstrate 2 orders of magnitude enhancement at photon energies up to $72 \mathrm{eV}$. Even higher intensities will barely be applicable with neutral gases. With our method, we already demonstrate the same enhancement of spectral intensity in the same spectral region in a pure proof-of-concept study at 1 order of magnitude lower driving intensity. This advantage is achieved by "targeted" trajectory steering following the guidelines of the "perfect wave" for HHG [21]. Furthermore, we can with good reason infer from our simulations that similar enhancement is possible at higher photon energies (Fig. 1) than those detected in our experiment (macroscopic phase matching provided). The enhancement will further improve when phase delay jitter is reduced by improving the laser amplifier CEP locking and actively stabilizing the multicolor interferometer. There is no principle obstacle in scaling our method towards much higher photon energies by increasing the intensities or adding the mid-IR idler wave, albeit technical challenges like phase matching at high photon energies and possibly shortening of the pulse envelopes [32] will have to be met. Finally, our simulations let us infer that our method has the important advantages of greatly increasing the periodicity of the attosecond pulse train (by a factor of 4, as compared to a factor of 2 in the case of Ref. [11]) and decreasing the attochirp (through the reduction of the cutoff excursion times, whereas these actually increase in the case of Ref. [11]).

Our demonstrated optimization holds significant potential for applications of HHG. Without sacrificing signal intensity for the self-probing of atoms and molecules [33], the desired higher bandwidths of the REWP can be attained even at moderate laser intensities, which is an important requirement for the study of larger molecules or clusters. The very dense harmonic spacing can be useful for higher spectral resolution in, e.g., determining the position of Cooper minima [27], dynamic or structural minima in HHG from aligned molecules [34], features due to resonances [35], and applications to high-resolution transient $\mathrm{x}$-ray absorption measurements. The corresponding large temporal spacing of the attosecond pulses is of interest in gating techniques for generating isolated attosecond pulses with high-energy multicycle drivers $[9,10,36]$. The reduced attochirp is highly advantageous for the generation of ever shorter and more intense attosecond pulses. It can be compensated with thinner, and thus less absorbing, metal filters [37], thereby further increasing the attainable attosecond pulse energy on target.

Our HHG enhancement results demonstrate a new example of the many possible applications of advanced shaping of optical cycles. Generally, any directly laserfield-driven process can be optimized by adequate intracycle pulse shaping. We expect new possibilities to emerge in a broad range of laser-matter interaction regimes, involving, e.g., Brunel electrons whose field-driven trajectories can lead to $\mathrm{THz}$ emission [38], plasma heating, and HHG on plasma mirrors [39], or particle acceleration [40].

\section{ACKNOWLEDGMENTS}

We thank B. Fabre for sharing with us his calculated argon recombination dipole. These studies were supported by the ERC (Projects No. CyFi 280202 and No. ASTEX 290467) and the EPSRC (Grants No. EP/I032517/1, No. EP/E028063/1, and No. EP/F034601/1). A.Z. acknowledges support from EPSRC Grant No. EP/J $002348 / 1$. S. H. acknowledges support by the EU-FP7IEF MUSCULAR and the Austrian Science fund (FWF) through Grant No. M1260-N16.

[1] P. B. Corkum, Plasma Perspective on Strong Field Multiphoton Ionization, Phys. Rev. Lett. 71, 1994 (1993).

[2] K. J. Schafer, B. Yang, L. F. DiMauro, and K. C. Kulander, Above Threshold Ionization beyond the High Harmonic Cutoff, Phys. Rev. Lett. 70, 1599 (1993). 
[3] A. Wirth, M. Th. Hassan, I. Grguraš, J. Gagnon, A. Moulet, T. T. Luu, S. Pabst, R. Santra, Z. A. Alahmed, A. M. Azzeer, V. S. Yakovlev, V. Pervak, F. Krausz, and E. Goulielmakis, Synthesized Light Transients, Science 334, 195 (2011).

[4] S.-W. Huang, G. Cirmi, J. Moses, K.-H. Hong, S. Bhardwaj, J. R. Birge, L.-J. Chen, E. Li, B. J. Eggleton, G. Cerullo, and F. X. Kärtner, High-Energy Pulse Synthesis with Sub-cycle Waveform Control for Strong-Field Physics, Nat. Photonics 5, 475 (2011).

[5] M. D. Perry and J. K. Crane, High-Order Harmonic Emission from Mixed Fields, Phys. Rev. A 48, R4051 (1993); S. Watanabe, K. Kondo, Y. Nabekawa, A. Sagisaka, and Y. Kobayashi, Two-Color Phase Control in Tunneling Ionization and Harmonic Generation by a Strong Laser Field and Its Third Harmonic, Phys. Rev. Lett. 73, 2692 (1994); H. Eichmann, A. Egbert, S. Nolte, C. Momma, B. Wellegehausen, W. Becker, S. Long, and J. K. McIver, Polarization-Dependent High-Order Two-Color Mixing, Phys. Rev. A 51, R3414 (1995).

[6] N. Dudovich, O. Smirnova, J. Levesque, Y. Mairesse, M. Y. Ivanov, D. M. Villeneuve, and P. B. Corkum, Measuring and Controlling the Birth of Attosecond XUV Pulses, Nat. Phys. 2, 781 (2006); J. M. Dahlström, A. L'Huillier, and J. Mauritsson, Quantum Mechanical Approach to Probing the Birth of Attosecond Pulses Using a Two-Colour Field, J. Phys. B 44, 095602 (2011).

[7] D. Shafir, H. Soifer, B. D. Bruner, M. Dagan, Y. Mairesse, S. Patchkovskii, M. Y. Ivanov, O. Smirnova, and N. Dudovich, Resolving the Time when an Electron Exits a Tunnelling Barrier, Nature (London) 485, 343 (2012).

[8] J. Mauritsson, P. Johnsson, E. Gustafsson, A. L'Huillier, K. J. Schafer, and M. B. Gaarde, Attosecond Pulse Trains Generated Using Two Color Laser Fields, Phys. Rev. Lett. 97, 013001 (2006).

[9] T. Pfeifer, L. Gallmann, M. J. Abel, D. M. Neumark, and S. R. Leone, Single Attosecond Pulse Generation in the Multicycle-Driver Regime by Adding a Weak SecondHarmonic Field, Opt. Lett. 31, 975 (2006).

[10] H. Mashiko, S. Gilbertson, C. Li, S. D. Khan, M. M. Shakya, E. Moon, and Z. Chang, Double Optical Gating of High-Order Harmonic Generation with CarrierEnvelope Phase Stabilized Lasers, Phys. Rev. Lett. 100, 103906 (2008).

[11] I. J. Jong Kim, C. M. Kim, H. T. Kim, G. H. Lee, Y. S. Lee, J. Y. Park, D. J. Cho, and C. H. Nam, Highly Efficient High-Harmonic Generation in an Orthogonally Polarized Two-Color Laser Field, Phys. Rev. Lett. 94, 243901 (2005).

[12] F. Brizuela, C. M. Heyl, P. Rudawski, D. Kroon, L. Rading, J. M. Dahlström, J. Mauritsson, P. Johnsson, C. L. Arnold, and A. L'Huillier, Efficient High-Order Harmonic Generation Boosted by Below-Threshold Harmonics, Sci. Rep. 3, 1410 (2013).

[13] R. Bartels, S. Backus, E. Zeek, L. Misoguti, G. Vdovin, I. P. Christov, M. M. Murnane, and H. C. Kapteyn, Shaped-Pulse Optimization of Coherent Emission of High-Harmonic Soft $X$ Rays, Nature (London) 406, 164 (2000); Y. Zheng, Z. Zeng, P. Zou, L. Zhang, X. Li, P. Liu, R. Li, and Z. Xu, Dynamic Chirp Control and Pulse Compression for Attosecond High-Order Harmonic Emission, Phys. Rev. Lett. 103, 043904 (2009).
[14] O. Raz, O. Pedatzur, B. D. Bruner, and N. Dudovich, Spectral Caustics in Attosecond Science, Nat. Photonics 6, 170 (2012).

[15] C. Vozzi, F. Calegari, F. Frassetto, L. Poletto, G. Sansone, P. Villoresi, M. Nisoli, S. De Silvestri, and S. Stagira, Coherent Continuum Generation above $100 \mathrm{eV}$ Driven by an IR Parametric Source in a Two-Color Scheme, Phys. Rev. A 79, 033842 (2009); T. Siegel, R. Torres, D. J. Hoffmann, L. Brugnera, I. Procino, A. Zaïr, J. G. Underwood, E. Springate, I. C. E. Turcu, L. E. Chipperfield, and J.P. Marangos, High Harmonic Emission from a Superposition of Multiple Unrelated Frequency Fields, Opt. Express 18, 6853 (2010).

[16] L. Brugnera, F. Frank, D. J. Hoffmann, R. Torres, T. Siegel, J. G. Underwood, E. Springate, C. Froud, E. I. C. Turcu, J. W. G. Tisch, and J. P. Marangos, Enhancement of High Harmonics Generated by Field Steering of Electrons in a Two-Color Orthogonally Polarized Laser Field, Opt. Lett. 35, 3994 (2010).

[17] M. Kitzler and M. Lezius, Spatial Control of Recollision Wave Packets with Attosecond Precision, Phys. Rev. Lett. 95, 253001 (2005).

[18] D. Shafir, Y. Mairesse, D. M. Villeneuve, P. B. Corkum, and N. Dudovich, Atomic Wave Functions Probed through Strong-Field Light-Matter Interaction, Nat. Phys. 5, 412 (2009).

[19] L. Brugnera, D. J. Hoffmann, T. Siegel, F. Frank, A. Zaïr, J. W. G. Tisch, and J. P. Marangos, Trajectory Selection in High Harmonic Generation by Controlling the Phase between Orthogonal Two-Color Fields, Phys. Rev. Lett. 107, 153902 (2011).

[20] T. Pfeifer, L. Gallmann, M. J. Abel, P. M. Nagel, D. M. Neumark, and S. R. Leone, Heterodyne Mixing of Laser Fields for Temporal Gating of High-Order Harmonic Generation, Phys. Rev. Lett. 97, 163901 (2006).

[21] L. E. Chipperfield, J. S. Robinson, J. W. G. Tisch, and J. P. Marangos, Ideal Waveform to Generate the Maximum Possible Electron Recollision Energy for Any Given Oscillation Period, Phys. Rev. Lett. 102, 063003 (2009).

[22] P. Wei, J. Miao, Z. Zeng, C. Li, X. Ge, R. Li, and Z. Xu, Selective Enhancement of a Single Harmonic Emission in a Driving Laser Field with Subcycle Waveform Control, Phys. Rev. Lett. 110, 233903 (2013).

[23] A. D. Shiner, C. Trallero-Herrero, N. Kajumba, H. C. Bandulet, D. Comtois, F. Légaré, M. Giguére, J. C. Kieffer, P. B. Corkum, and D. M. Villeneuve, Wavelength Scaling of High Harmonic Generation Efficiency, Phys. Rev. Lett. 103, 073902 (2009).

[24] See Supplemental Material at http://link.aps.org/ supplemental/10.1103/PhysRevX.4.021028 for technical details on the experimental setup and the simulations, as well as additional supporting simulations and measurements.

[25] T. Popmintchev, M.-C. Chen, D. Popmintchev, P. Arpin, S. Brown, S. Ališauskas, G. Andriukaitis, T. Balčiunas, O. D. Mücke, A. Pugzlys, A. Baltuška, B. Shim, S. E. Schrauth, A. Gaeta, C. Hernández-García, L. Plaja, A. Becker, A. Jaron-Becker, M. M. Murnane, and H. C. Kapteyn, Bright Coherent Ultrahigh Harmonics in the keV X-Ray Regime 
from Mid-Infrared femtosecond Lasers, Science 336, 1287 (2012).

[26] A. Baltuška, T. Fuji, and T. Kobayashi, Controlling the Carrier-Envelope Phase of Ultrashort Light Pulses with Optical Parametric Amplifiers, Phys. Rev. Lett. 88, 133901 (2002).

[27] J. Higuet, H. Ruf, N. Thiré, R. Cireasa, E. Constant, E. Cormier, D. Descamps, E. Mével, S. Petit, B. Pons, Y. Mairesse, and B. Fabre, High-Order Harmonic Spectroscopy of the Cooper Minimum in Argon: Experimental and Theoretical Study, Phys. Rev. A 83, 053401 (2011).

[28] A. T. Le, R. R. Lucchese, S. Tonzani, T. Morishita, and C. D. Lin, Quantitative Rescattering Theory for High-Order Harmonic Generation from Molecules, Phys. Rev. A 80, 013401 (2009).

[29] M. Lewenstein, Ph. Balcou, M. Y. Ivanov, A. L'Huillier, and P. B. Corkum, Theory of High-Harmonic Generation by Low-Frequency Laser Fields, Phys. Rev. A 49, 2117 (1994).

[30] G. Sansone, C. Vozzi, S. Stagira, and M. Nisoli, Nonadiabatic Quantum Path Analysis of High-Order Harmonic Generation: Role of the Carrier-Envelope Phase on Short and Long Paths, Phys. Rev. A 70, 013411 (2004).

[31] We apply the same genetic algorithm as in Ref. [21] to find the optimal combination (relative fluences and phase delays) of our available three colors. This led to an optimal wave with $64 \%$ of the total fluence in the $1.5-\mu \mathrm{m}$ component, $28 \%$ in the $1.0-\mu \mathrm{m}$ component, $8 \%$ in the $0.5-\mu \mathrm{m}$ component, and $\phi_{\mathrm{CEP}}=0.85 \pi, \phi_{2}=0.12 \pi$. Experimentally, we have to make a compromise between a high relative weight of the 1.5- $\mu \mathrm{m}$ component and a high total pulse energy (the energy in the $1.5-\mu \mathrm{m}$ pulse is limited). We, therefore, limit the relative fluence of the $1.5-\mu \mathrm{m}$ component to $\approx 50 \%$. Also, the relative fluence of the $0.5-\mu \mathrm{m}$ component is only $\approx 2.5 \%$ in our experiments. Fixing these relative fluences and running the optimization algorithm to find the optimal phase delays leads to the waveform with $\phi_{\mathrm{CEP}}=0.85 \pi$, $\phi_{2}=0.2 \pi$, already shown in Fig. 1 . Simulations [24] show that this waveform, which is within the parameter space of our current setup, can be expected, on the single-atom level, to lead to an only slightly lower spectral cutoff and spectral intensities lower only by a factor $<3$ as compared to the "perfect wave" from Ref. [21].
[32] G. Andriukaitis, D. Kartashov, D. Lorenc, A. Pugžlys, A. Baltuška, L. Giniunas, R. Danielius, J. Limpert, T. Clausnitzer, E. B. Kley, A. Voronin, and A. Zheltikov, Hollow-Fiber Compression of $6 \mathrm{~mJ}$ Pulses from a Continuous-Wave Diode-Pumped Single-Stage $\mathrm{Yb}, \mathrm{Na}: \mathrm{CaF}_{2}$ Chirped Pulse Amplifier, Opt. Lett. 36, 1914 (2011).

[33] S. Haessler, J. Caillat, and P. Salières, Self-Probing of Molecules with High Harmonic Generation, J. Phys. B 44, 203001 (2011).

[34] R. Torres, T. Siegel, L. Brugnera, I. Procino, J. G. Underwood, C. Altucci, R. Velotta, E. Springate, C. Froud, I. C. E. Turcu, S. Patchkovskii, M. Y. Ivanov, O. Smirnova, and J. P. Marangos, Revealing Molecular Structure and Dynamics through High-Order Harmonic Generation Driven by Mid-IR Fields, Phys. Rev. A 81, 051802 (2010).

[35] S. Haessler, V. Strelkov, L. B. Elouga Bom, M. Khokhlova, O. Gobert, J. F. Hergott, F. Lepetit, M. Perdrix, T. Ozaki, and P. Salières, Phase Distortions of Attosecond Pulses Produced by Resonance-Enhanced High Harmonic Generation, New J. Phys. 15, 013051 (2013).

[36] E. J. Takahashi, P. Lan, O. D. Mücke, Y. Nabekawa, and K. Midorikawa, Attosecond Nonlinear Optics Using Gigawatt-Scale Isolated Attosecond Pulses, Nat. Commun. 4, 2691 (2013).

[37] R. Lopez-Martens, K. Varjú, P. Johnsson, J. Mauritsson, Y. Mairesse, P. Salières, M. B. Gaarde, K. J. Schafer, A. Persson, S. Svanberg, C. G. Wahlström, and A. L'Huillier, Amplitude and Phase Control of Attosecond Light Pulses, Phys. Rev. Lett. 94, 033001 (2005).

[38] M. Kress, T. Loffler, M. D. Thomson, R. Dorner, H. Gimpel, K. Zrost, T. Ergler, R. Moshammer, U. Morgner, J. Ullrich, and H. G. Roskos, Determination of the Carrier-Envelope Phase of Few-Cycle Laser Pulses with Terahertz-Emission Spectroscopy, Nat. Phys. 2, 327 (2006).

[39] F. Quéré, C. Thaury, P. Monot, S. Dobosz, J. P. Geindre, and P. Audebert, Coherent Wake Emission of High-Order Harmonics from Overdense Plasmas, Phys. Rev. Lett. 96, 125004 (2006).

[40] M. Veltcheva, A. Borot, C. Thaury, A. Malvache, E. Lefebvre, A. Flacco, R. Lopez-Martens, and V. Malka, Brunel-Dominated Proton Acceleration with a Few-Cycle Laser Pulse, Phys. Rev. Lett. 108, 075004 (2012). 